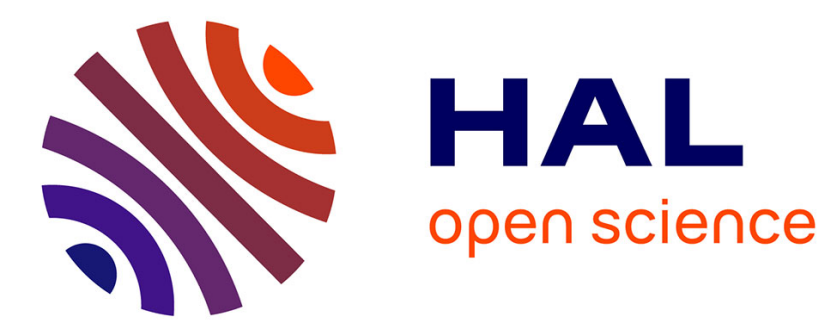

\title{
Étude du contrôle du décollement sur un profil d'aile par mesures PIV et analyse POD \\ Julien Favier, Azeddine Kourta
}

\section{To cite this version:}

Julien Favier, Azeddine Kourta. Étude du contrôle du décollement sur un profil d'aile par mesures PIV et analyse POD. Comptes rendus de l'Académie des sciences. Série IIb, Mécanique, 2006, 334, pp.272-278. 10.1016/j.crme.2006.02.005 . hal-01073970

\section{HAL Id: hal-01073970 https://hal.science/hal-01073970}

Submitted on 11 Oct 2014

HAL is a multi-disciplinary open access archive for the deposit and dissemination of scientific research documents, whether they are published or not. The documents may come from teaching and research institutions in France or abroad, or from public or private research centers.
L'archive ouverte pluridisciplinaire HAL, est destinée au dépôt et à la diffusion de documents scientifiques de niveau recherche, publiés ou non, émanant des établissements d'enseignement et de recherche français ou étrangers, des laboratoires publics ou privés. 


\title{
Étude du contrôle du décollement sur un profil d'aile par mesures PIV et analyse POD
}

\author{
Julien FAVIER Azeddine KOURTA \\ Institut de Mécanique des Fluides de Toulouse, Allée du professeur Camille Soula, \\ 31400 Toulouse, France
}

Reçu le ${ }^{* * * * *}$; accepté après révision le +++++

\section{Résumé}

L'objet de l'étude concerne le contrôle actif du décollement de la couche limite autour d'un profil ONERA D, à l'aide de microjets à soufflage continu. La vélocimétrie laser par images de particules (PIV) est mise en œuvre pour mesurer les champs de vitesse de la demi-envergure du profil dans les cas d'écoulements contrôlés et non contrôlés. Un post-traitement par décomposition en modes propres orthogonaux (POD) est développé pour mettre en évidence les caractéristiques énergétiques principales de l'écoulement. Ainsi, l'étude ciblée des modes POD dans des configurations d'écoulement décollé ou décroché permet de mieux appréhender les mécanismes de contrôle. De plus la comparaison des bases POD obtenues dans les cas contrôlés et non contrôlés ouvre la voie à des méthodes d'optimisation des stratégies de contrôle. Pour citer cet article: A. Nom1, A. Nom2, C. R. Mecanique 333 (2005). 


\section{Abstract}

\section{Study of separation control on a wing profile using PIV measurements}

and POD analysis. The purpose of this study is the active control of flow separation on an ONERA D airfoil, using continuous blowing microjets. Particle Image Velocimetry (PIV) is used to measure velocity fields at midspan around the airfoil in both controlled and uncontrolled cases. Post-processing based on Proper Orthogonal Decomposition (POD) is developped to exhibit the main energetic features of the flow. Therefore, the detailed study of the POD modes for separated and stalled cases leads to a better understanding of the control mechanisms. Moreover, the POD basis computed for uncontrolled and controlled cases, opens the way to optimisation of control strategies. To cite this article: A. Nom1, A. Nom2, C. R. Mecanique 333 (2005).

Key words: Control of separation; PIV; POD

Mots-clés : Contrôle du décollement; PIV; POD

\section{Introduction}

Le contrôle du décollement de la couche limite autour d'un profil trouve des applications nombreuses et variées dans diverses configurations aéronautiques car l'aptitude à supprimer ou retarder le phénomène de décollement conduit à améliorer le niveau de portance, réduire le bruit et la traînée générée sur les engins aéronautiques, ce qui représente des enjeux économiques et technologiques évidents. De nombreuses études ont jusqu'ici été menées sur le

Email addresses: favier@imft.fr (Julien FAVIER), kourta@imft.fr (Azeddine KOURTA). 
plan expérimental pour tester l'efficacité de différents actionneurs capables de contrôler le décollement et les différents procédés d'action sont extrêmement diversifiés. Par exemple, en ce qui concerne les méthodes dites "actives" qui introduisent de l'énergie extérieure dans le fluide, on distingue l'utilisation des parois mobiles [1], des méthodes à base d'aspiration ou de soufflage [2,3], des méthodes acoustiques [4], thermiques [5] ou électromagnétiques [6]. Cette liste est loin d'être exhaustive et plusieurs de ces travaux offrent des résulats excellents et des perspectives très prometteuses. Dans le cadre de la présente étude, un contrôle actif à base de soufflage a été mis en œuvre en utilisant des microjets à soufflage continu, qui constituent des générateurs fluidiques de tourbillons. Cependant, il apparait nécessaire de connaître a priori la meilleure action qui doit être appliquée à un écoulement donné pour supprimer ou retarder le décollement et concevoir ainsi un actionneur adapté aux besoins du contrôle. C'est le rôle des méthodes d'optimisation qui recherchent la meilleure action à appliquer au fluide pour atteindre l'objectif de réduire efficacement le décollement en utilisant les équations de comportement du fluide qui sont celles de Navier-Stokes. Afin de réduire les coûts numériques de ces méthodes qui sont souvent prohibitifs pour des configurations complexes, une méthode consiste à approcher le système d'équations de Navier-Stokes par un modèle d'ordre réduit basé sur la décomposition orthogonale en modes propres (POD) [7]. Le modèle est construit par projection des équations de Navier-Stokes sur les modes POD et il est alors possible de ne prendre en compte que quelques modes pour avoir un modèle d'ordre bas et ainsi réduire considérablement les couts de calcul liés aux méthodes d'optimisation telles que les méthodes de contrôle optimal par exemple[7,8]. Dans cette étude, le contrôle du décollement de la couche limite est effectué de manière expérimentale sur un profil d'aile symétrique ONERA D à l'aide d'un action- 
neur actif de soufflage précédemment utilisés lors de travaux antérieurs [9] et qui permet des gains de portance de l'ordre de $20 \%$ dans des configurations décrochées. L'efficacité du contrôle est mis en évidence et les caratéristiques des modes POD sont discutées dans les cas controlés et non contrôlés afin de mieux cerner les mécanismes physiques mis en jeu par le contrôle.

\section{Dispositif expérimental et métrologie}

\subsection{Protocole expérimental}

La maquette du profil ONERA D de $0.35 \mathrm{~m}$ de corde et $1 \mathrm{~m}$ d'envergure est montée entre plaques de garde dans la veine d'essai de la soufflerie à veine ouverte S1 de l'IMFT. Les actionneurs sont installés à l'intérieur de la maquette, grâce à un capot d'extrados amovible (Fig. 1) et sont situés sur l'envergure du capot. Un soufflage continu par l'intermédaire de 82 micro-orifices de 0.4 mm de diamètre usinés dans le profil, au niveau de la zone de décollement et le long d'une ligne parallèle au bord d'attaque. La zone de contrôle représente $40 \%$ de l'envergure du profil. Le dispositif nécessaire au fonctionnement des actionneurs est installé sous le capot, dans la région proche du bord d'attaque (cf [9] pour une description plus détaillée du dispositif expérimental). Cette technique a été développée en collaboration avec F.S. Alvi qui a effectué de nombreux travaux sur ce type d'actionneur et qui a contribué à son implémentation et à son étude sur le profil [10]. Par l'intermédiaire de ces orifices, le soufflage se fait perpendiculairement à la corde et la vitesse des jets est sonique. La vitesse est importante en raison de la très petite section des microjets et 2 bars d'air comprimé sont nécessaires pour obtenir un tel 
soufflage [9].

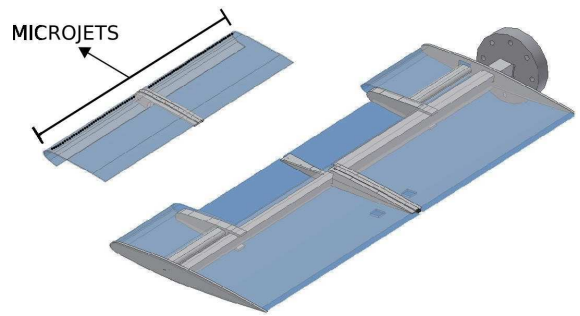

a) Capot d'extrados amovible

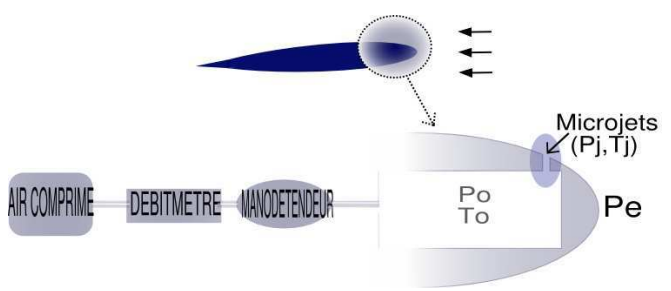

b) Actionneurs de contrôle

FiG. 1. Dispositif expérimental

\subsection{Mesures PIV}

La PIV est utilisée pour mesurer des champs de vitesse bidimensionnels pour différentes valeurs de nombre de Reynolds, d'incidence, avec et sans contrôle (Fig. 2).

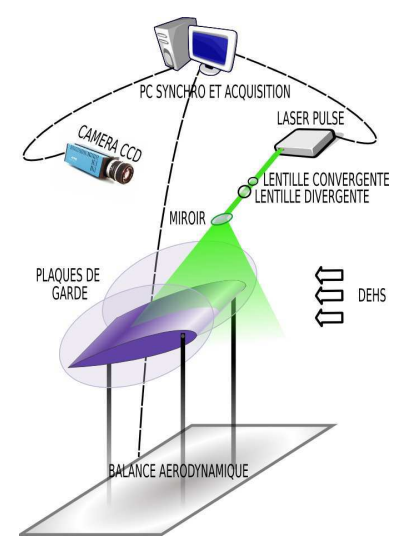

FIG. 2. Protocole expérimental

L'écoulement est ensemencé par des particules de DEHS calibrées à $1 \mu \mathrm{m}$ de diamètre. Les images de l'écoulement sont réalisées avec une caméra CCD PCO 2000 fixée sur une table rotative afin de suivre les variations d'angle d'attaque du profil. La caméra de résolution 2048 × 2048 pixels est équipée d'un objectif de $85 \mathrm{~mm}$ avec une ouverture de diaphragme de 8 . 
Le laser utilisé est pulsé de type Nd-YAG Quantel $(2 \times 200$ mJ $)$ et l'intervalle de temps entre deux flashs est fixé à $20 \mu$ s ou $15 \mu$ s selon la vitesse de l'écoulement amont. Les acquisitions de données se font à une fréquence de $10 \mathrm{~Hz}$, la caméra et le laser étant synchronisés par un PC.

Les mesures sont effectuées à mi-envergure et le profil est équipé de plaques de garde de façon à obtenir un champ bidimensionnel le long de la corde. Deux zones sont explorées, une près du bord d'attaque et une plus en aval, près du bord de fuite. Pour chaque configuration d'angle d'attaque, de nombre de Reynolds et chaque zone de mesure, 1000 paires d'images sont enregistrées, avec une résolution spatiale de $2048 \times 2048$ pixels et une dynamique de 16000 niveaux de gris. Trois valeurs de l'angle d'incidence ont été testés, à savoir $\alpha=0^{\circ}$ pour le cas non décollé, $\alpha=13^{\circ}$ pour une configuration de couche limite décollée et $\alpha=16^{\circ}$ pour le cas décroché, le tout pour trois nombres de Reynolds, $\mathcal{R}_{e}=0.3510^{6}, \mathcal{R}_{e}=0.4610^{6}$ et $\mathcal{R}_{e}=0.610^{6}$, avec et sans contrôle.

\subsection{Traitement d'images}

Après fenêtrage, les images de taille $2048 \times 1400$ pixels sont analysées par un algorithme de corrélation croisé implémenté dans le logiciel PIVIS développé à l'IMFT (service signaux et images). Cet algorithme est basé sur une FFT bidimensionnelle des fonctions de corrélation et utilise un schéma itératif avec déformation d'image sub-pixel [11]. De plus, l'écoulement est analysé par recouvrement de cellules d'interrogation à 50\% afin d'améliorer la résolution spatiale des champs de vitesse. Les images sont subdivisées en fenêtres d'interrogation rectangulaires de taille $16 \times 16$ pixels, c'est-à-dire que la plus petite 
fenêtre est de taille $2.3 \times 2.3 \mathrm{~mm}$ dans un champ de $30 \times 30 \mathrm{~cm}$. Une attention particulière a été accordée à la résolution du champ afin d'obtenir un maillage suffisamment fin pour identifier les effets des microjets près du bord d'attaque. Après une moyenne sur 1000 champs de vecteurs, les calculs de corrélations sont convergés et le besoin d'interpolation est minimal.

\section{Résultats PIV}

La figure 3 présente un champ de vitesse moyenne dans une configuration décrochée, $\alpha=16^{\circ}$ et $R_{e}=4.6710^{5}$. Grâce aux deux zones mesurées, des données sont ainsi obtenues sur la quasi-totalité de la corde (cf Fig. 3). La couche limite décolle complètement sur l'extrados. La zone du bord de fuite n'est pas représentée sur le graphe car la puissance du laser y est plus faible et la qualité des corrélations insuffisante. Sur la figure 3, un fort décollement est visible dans la zone en bleu foncé qui représente les vitesses négatives de la couche limite décollée. La zone d'ombre de mesure près de la paroi est représentée en noir sur le graphe et possède une épaisseur d'environ $5 \mathrm{~mm}$. La couche limite décolle sur ce profil à environ $5 \%$ en corde du bord d'attaque.

La figure 4 présente un exemple des champs moyens bidimensionnels obtenus par PIV dans le cas d'une configuration décrochée $\left(\alpha=16^{\circ}\right.$ et $\left.R_{e}=0.4610^{6}\right)$. L'effet du contrôle est mis en évidence par la disparition de la zone décollée sur les profils de vitesse (Fig. 5) ou sur les contours de champs de vitesse moyenne (Fig. 4), ce qui est à mettre en relation directe avec les gains de portance de $20 \%$ obtenus avec le contrôle par microjets [9]. La modification de l'écoulement au voisinage du bord d'attaque est clairement mise en évidence dans le cas du soufflage par microjets et se manifeste par une gerbe de survitesse au dessus 


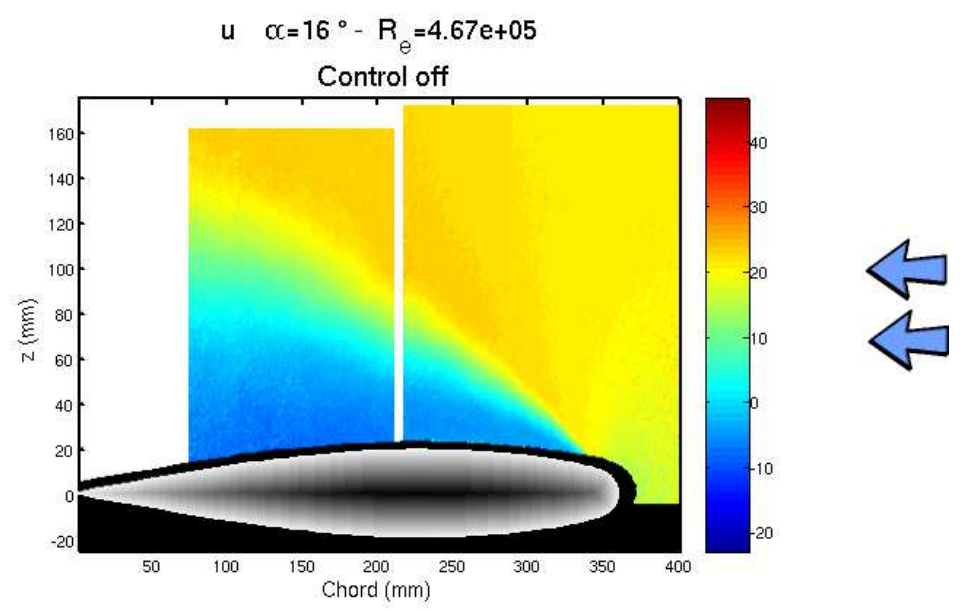

FiG. 3. Champ de vitesse moyenne en configuration décrochée

de la zone de contrôle (Fig. 4).

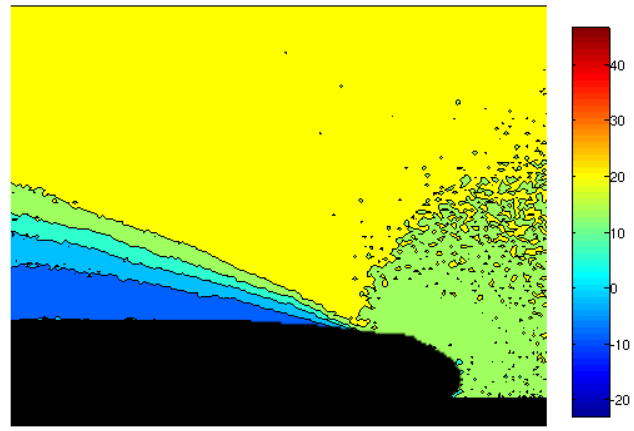

a) Sans contrôle

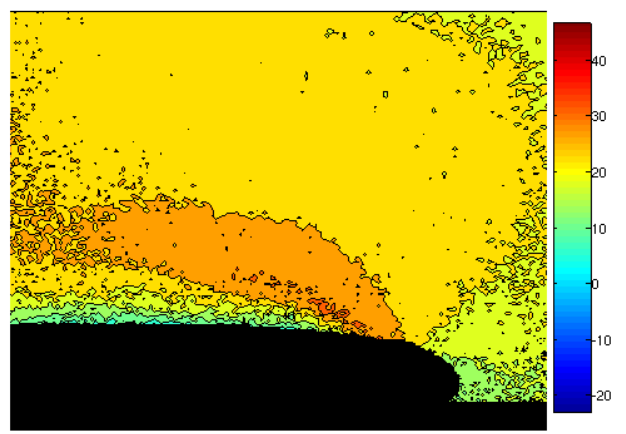

b) Avec contrôle

Fig. 4. Contours de champs de vitesse moyenne dans la zone proche du bord d'attaque pour $\mathcal{R}_{e}=0.4610^{6}$ et $\alpha=16^{o}$ 


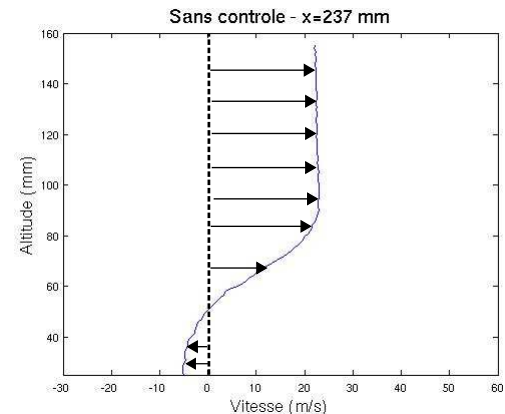

a) Sans contrôle

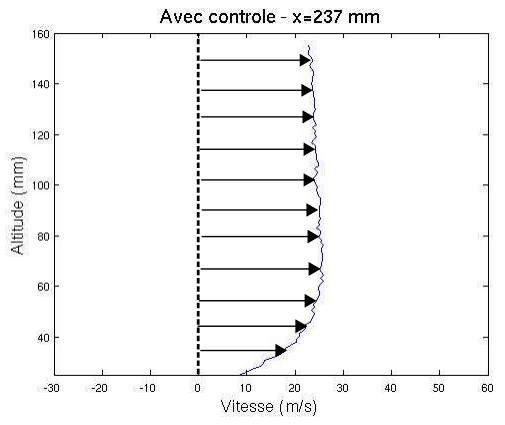

b) Avec contrôle

Fig. 5. Profils de vitesse à $33 \%$ de la corde pour $\mathcal{R}_{e}=0.4610^{6}$ et $\alpha=16^{\circ}$

\section{Décomposition en modes POD}

\subsection{Principe}

Dans le cadre de cette étude, la décomposition en modes propres (POD) utilisée est celle introduite par Sirovich [12]. Cette approche, appelée "méthode des snapshots", a la particularité de séparer le temps et l'espace et s'avère être très intéressante en terme de temps de calcul sur des bases de données de type PIV, ou sur des champs de vitesse simulés numériquement où la finesse du maillage spatial est importante devant la discrétisation temporelle des "snapshots". Au terme de la décomposition, la vitesse bidimensionnelle $\mathbf{u}(x, y, t)$ s'écrit :

$$
u^{i}(x, y, t)=\sum_{k=1}^{N} a_{k}(t) \Phi_{k}^{i}(x, y)
$$

A partir de la vitesse fonction du temps et de l'espace $\mathbf{u}(x, y, t)$, on cherche une base discrète de fonctions de l'espace $\boldsymbol{\Phi}(x, y)$ appelés modes propres qui vont approcher "au mieux" en moyenne la vitesse $\mathbf{u}(x, y, t)$. D'un point de vue mathématique, on cherche à maximiser :

$$
\frac{<(\mathbf{u}, \Phi)^{2}>}{(\Phi, \Phi)}
$$


où $<$. $>$ est la moyenne en temps et $(.,$.$) est un produit scalaire.$

Dans le cas de la méthode des snapshots, cette maximisation peut être reformulée par le problème aux valeurs propres suivant : $\underline{C} a=\lambda a$ ( $\mathrm{cf}[13]$ pour une démonstration détaillée). Les $\lambda_{k}$ sont les valeurs propres et $\underline{C}$ est la matrice de corrélation temporelle définie par : $\underline{C}\left(t, t^{\prime}\right)=<u_{t}(x, y) u_{t^{\prime}}(x, y)>$. Conformément à la méthode des "snapshots", il s'agit d'une corrélation en temps faite sur les champs de vitesse. Les valeurs propres $\lambda_{k}$ correspondent à l'énergie du mode $k$ et les vecteurs propres associés $a_{k}$ sont appelés les modes temporels. Les modes spatiaux sont ensuite déduits par projection : $\boldsymbol{\Phi}_{k}(x, y)=\sum_{k=1}^{N_{t}} a_{k} \mathbf{u}_{k}(x, y)$.

L' équation 1 permet alors de reconstruire les champs de vitesse sur un nombre fini $N$ de modes qui est choisi en fonction du niveau d'énergie que l'on désire représenter. Afin de mettre en évidence les structures cohérentes présentes dans la couche limite, la décomposition en mode POD a été effectuée sur la vitesse fluctuante définie par $\mathbf{u}^{\prime}=\mathbf{u}-<\mathbf{u}>$.

\subsection{Analyse des modes}

Pour explorer la configuration décrochée $\mathcal{R}_{e}=0.4610^{6}$ et $\alpha=16^{\circ}$, l'analyse des modes POD sera effectuée avec et sans contrôle. Le premier mode de la composante longitudinale de la vitesse fluctuante $\mathbf{u}^{\prime}$ est représenté sur la figure 6. La forme de ce mode est différente suivant que l'on s'intéresse à la configuration contrôlée ou non. Cette forme correspond à une structure de l'ordre de grandeur de la zone décollée pour le cas sans contrôle. Dans le cas du contrôle, elle est de l'ordre de grandeur du jet des actionneurs. L'énergie contenue dans chaque mode est donnée dans les deux cas sur la figure 7. Cette 


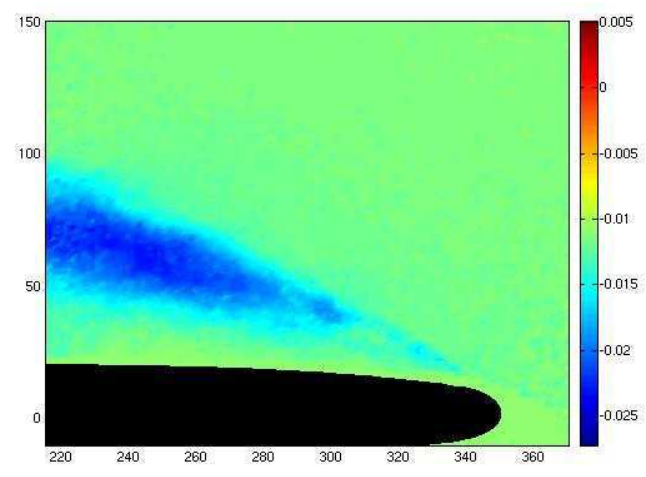

a) Sans contrôle

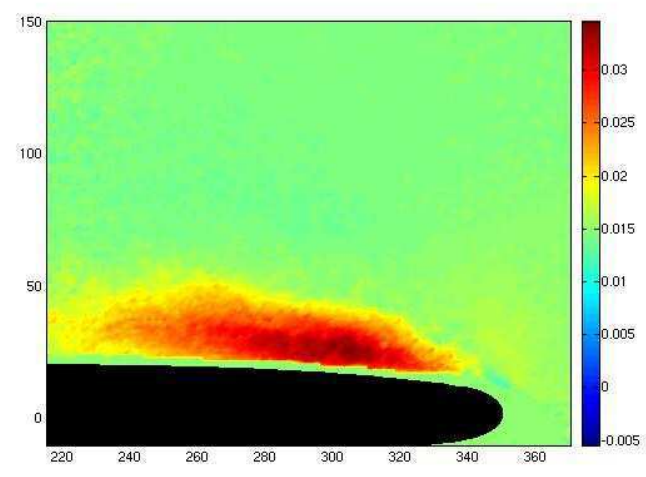

b) Avec contrôle

Fig. 6. Premier mode pour $\mathcal{R}_{e}=0.4610^{6}$ et $\alpha=16^{\circ}$

courbe représente la décroissance des valeurs propres en fonction du numéro du mode. Ainsi le premier mode contient 15\% de l'énergie de l'écoulement dans les cas sans contrôle et $49 \%$ dans le cas du contrôle. Un résultat important est que l'action du contrôle se retouve énergétiquement sur le premier mode uniquement, les modes suivants ayant sensiblement les mêmes contributions énergétiques par rapport à l'énergie totale. L'influence du premier mode est donc prépondérante dans la mise en œuvre d'une méthode de contrôle optimal. Dans cette configuration de décrochage sans contrôle, $50 \%$ de l'énergie est contenue dans 10 modes, ce qui montre que les premiers modes ne dominent pas complètement la dynamique. Ainsi, pour reconstruire par exemple $99 \%$ de l'énergie de l'écoulement à l'aide de l'équation 1, il est nécessaire de prendre en compte 150 modes. Le cas contrôlé, dont la dynamique est moins complexe puisque la zone décollée est supprimée, est moins coûteux à reconstruire puisque le premier mode représente quasiment la moitié de l'énergie totale. Ainsi, il apparait que l'effet du contrôle se manifeste principalement par une structure de grande échelle (Fig. 6) qui est de l'ordre de grandeur de l'épaisseur de couche limite décollée. Le mécanisme qui correspond à cette structure spa- 
tiale du premier mode tient au fait que les microjets génèrent des tourbillons qui sont advectés le long de la corde, dans le sens de l'écoulement. En effet, chaque jet normal par rapport à l'écoulement transverse de la soufflerie produit en aval des perturbations en forme de paires de tourbillons contra-rotatifs [14]. Ces tourbillons ont un effet stabilisateur pour le phénomène de décollement car ils mélangent le fluide fortement ralenti en proche paroi avec les couches fluides plus rapides de la zone de mélange. Cette ré-énergisation de la couche limite permet de la rendre plus résistante au décollement et favorise donc la supression de ce phénomène pénalisant pour les performances aérodynamiques d'un profil.

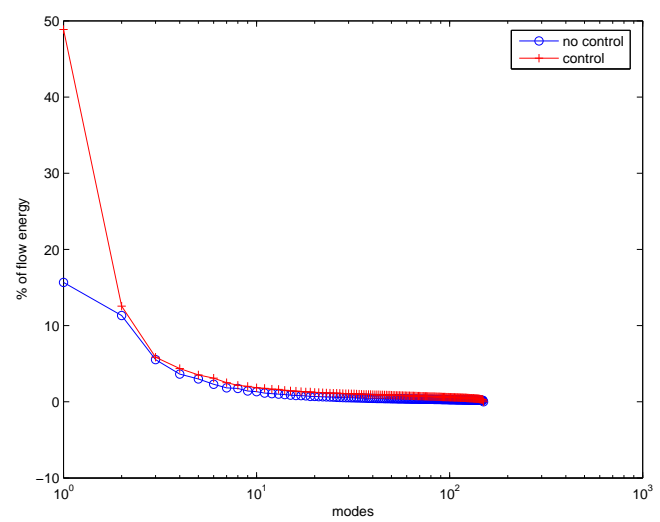

FIG. 7. Energie relative à chaque mode pour $\mathcal{R}_{e}=0.4610^{6}$ et $\alpha=16^{\circ}$

Les modes suivants sont de moins en moins énergétiques et correspondent à des structures de plus petite taille, qui traduisent les traces du passage de structures cohérentes dans la couche limite, comme on peut l'observer sur le deuxième mode par exemple (Fig.8). 


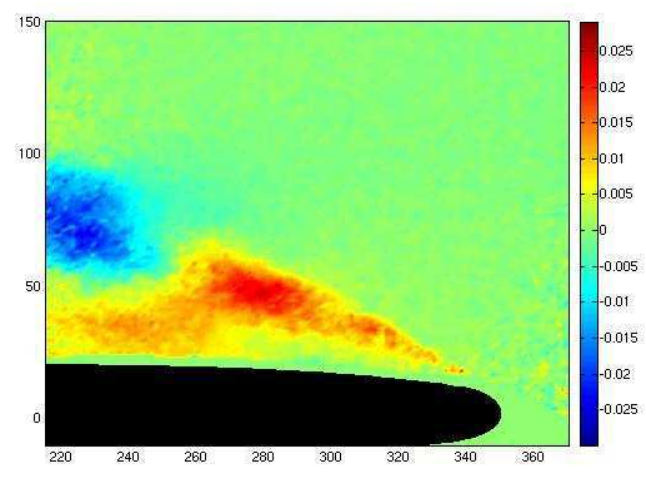

a) Mode 2 sans contrôle

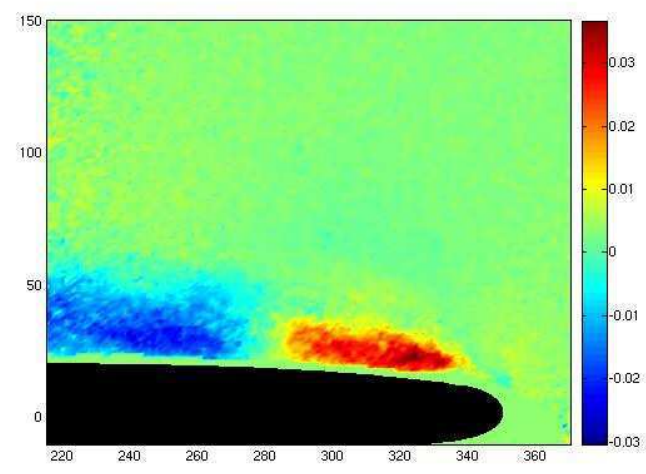

b) Mode 2 avec contrôle

Fig. 8. Deuxième mode pour $\mathcal{R}_{e}=0.4610^{6}$ et $\alpha=16^{\circ}$

\section{Conclusions et perspectives}

- L'analyse des champs PIV a permis de montrer l'efficacité réelle du contrôle par microjets dans la suppression de la zone de décollement, dans une configurations de décrochage.

- Grâce à la décomposition en modes POD, le mécanisme de recollement de la couche limite par redistribution de l'énergie est mis en évidence. Le premier mode se révèle déterminant dans l'application de méthodes de contrôle optimal car il est lié au mécanisme de recollement.

- La base POD obtenue ici empiriquement va permettre de construire un système dynamique par projection de Galerkin des équations de NavierStokes sur les modes POD. En ne retenant qu'un nombre réduit de modes, le modèle d'ordre réduit ainsi créé rend possible l'application de méthodes de contrôle optimal car il réduit considérablement les temps de calcul imposés par les boucles d'optimisation. L'action de contrôle optimale pour réduire le décollement pourra alors être finalement obtenue pour ce modèle d'ordre réduit et testée expérimentalement sur le profil d'aile. 


\section{Remerciements}

Les auteurs tiennent à remercier Sébastien Cazin et Emmanuel Cid du service "Signaux et Images" de l'IMFT pour leur aide dans la réalisation des mesures PIV. Ces travaux s'inscrivent dans le cadre du GDR2502 "Contrôle des décollements".

\section{Références}

[1] V. J. Modi, F. Mokhtarian, M. Fernando, and T. Yokozimo. Moving surface boundary layer control as applied to 2-d airfoils. AIAA paper, pages 89-0296, 1989.

[2] I. Wygnanski. Boundary layer flow control by periodic addition of momentum. In 4th AIAA Shear flow control conference, Silvertree hotel Snowmass village, CO, june 29-July 21997.

[3] D.C. McCormick. Boundary layer separation control with directed synthetic jets. AIAA paper, 2000-0519, January 2000.

[4] F.G. Collins. Boundary layer control on wings using sound and leading edge serrations. AIAA, pages 1979-1875, 1979.

[5] P. K. Chang. Control of flow separation. Hemisphere, Washington, D. C., 1976.

[6] M. Gad-el-Hak. Flow Control, passive, active and reactive management. Cambridge University Press, 2000.

[7] M. Bergmann, L. Cordier, and J.P. Brancher. Optimal rotary control of the cylinder wake using proper orthogonal decomposition reduced order-model. Physics of Fluids, 17, 2005. 
[8] B. Galletti, A. Bottaro, C.H. Bruneau, and A. Iollo. Accurate model reduction of transcient flows. European journal of Mechanics B / Fluids, 2005.

[9] J. Favier, S. Bourgois, E. Sommier, J. Tensi, and A. Kourta. Contrôle fluidique du décollement sur un profil d'aile. In 17 ème Congrès Français de Mécanique, Troyes, France, septembre 2005.

[10] K. A. Phalnikar, F.S. Alvi, and C. Shih. Behavior of free impinging supersonic microjets. AIAA paper, 2001-3047, 2001.

[11] B. Lecordier and M. Trinite. Advanced piv algorithms with image distortion validation and comparison from synthetic images of turbulent flow. In PIV03 Symposium, Busan, Korea, 2003.

[12] L. Sirovich. Turbulence and the dynamics of coherent structures. Quaterly of Applied Mathematics, XLV, 3 :561-590, 1987.

[13] L. Cordier and M.Bergmann. Proper orthogonal decomposition : an overview. In Lecture series 2002-04 on post-processing of experimental and numerical data, Von Kármán Institute of Fluid Dynamics, 2002.

[14] T. H. New and J. Soria. Near-field pairing of leading-edge vortices in elliptic jets in cross flow. European Journal of Mechanics B/Fluids, 23 :551-569, 2004.

[15] S. Bougois, F.S. Alvi, J. Tensi, and J.P. Bonnet. Control of flow separation using microjets. In First European Forum on Flow Control, Poitiers, France, October 11-14, 2004. 